\title{
Intranasal delivery of recombinant MANF protein is neuroprotective in cortical ischemia-reperfusion injury in rats
}

\author{
Jenni E Anttila ${ }^{1}$, Kert Mätlik ${ }^{1}$, Olli S Mattila², Päivi Lindholm ${ }^{1}$, Perttu J Lindsberg ${ }^{2}$, Mikko Airavaara ${ }^{1}$ \\ ${ }^{1}$ Institute of Biotechnology, University of Helsinki, Finland, ${ }^{2}$ Research Program for Molecular Neurology, University of \\ Helsinki, Finland
}

Background: Stroke is one of the leading causes of death and disability worldwide but the treatment options remain limited. Mesencephalic astrocyte-derived neurotrophic factor (MANF) is an $18 \mathrm{kDa}$ endoplasmic reticulum (ER) resident protein that is secreted upon ER stress and known to be upregulated in the brain after ischemia. MANF has neurotrophic properties and intracranial delivery of recombinant MANF is neuroprotective in rat models of ischemic stroke. Our aim was to test if non-invasive intranasal delivery of MANF is neuroprotective and determine the pharmacokinetic profile of MANF after intranasal administration.

Methods: Unilateral cortical infarction was induced in adult male Sprague Dawley rats by transiently ligating the distal branch of the right middle cerebral artery with a 10-0 suture and occluding both common carotid arteries for 60 minutes. Recombinant human MANF (rhMANF) or vehicle was administered intranasally before the induction of ischemia and after reperfusion. Effects on infarct volume were measured $48 \mathrm{~h}$ post-stroke $(\mathrm{n}=10-17)$. In separate experiments, behavioral recovery was monitored for 14 days with neurologigal tests and locomotor activity $(\mathrm{n}=14-15)$. The effect of rhMANF on ischemic and post-ischemic cerebral blood flow $(\mathrm{CBF})$ was evaluated with Laser Doppler Flowmetry $(n=9$ -10). The distribution of rhMANF was investigated using I125-rhMANF and an enzyme-linked immunosorbent assay (ELISA; $n=6)$. ELISA was used also to measure endogenous MANF levels in sera after cortical ischemia $(n=3-6)$.

Results: Intranasally administered rhMANF decreased infarction volume by $30 \%(\mathrm{p}<0.05)$ and reduced neurological deficits measured on days $7(\mathrm{p}<0.05)$ and $14(\mathrm{p}<0.0001)$ post-stroke. No significant differences were found between the treatment groups in $\mathrm{CBF}$ during occlusion or reperfusion. One hour after intranasal delivery $0.4 \%$ of the total dose of I125-rhMANF reached systemic circulation and $0.003 \%$ was detected in the brain. Endogenous MANF serum levels were not altered after cerebral ischemia.

Conclusions: Our results show that rhMANF is neuroprotective also when administered intranasally, providing reduced infarct volumes and improved functional recovery. We also show that endogenous levels of MANF in the systemic circulation are not affected by cerebral ischemia. Further investigation is warranted to determine the individual contribution of circulating and intracerebral rhMANF in the neuroprotective effects after intranasal administration. 